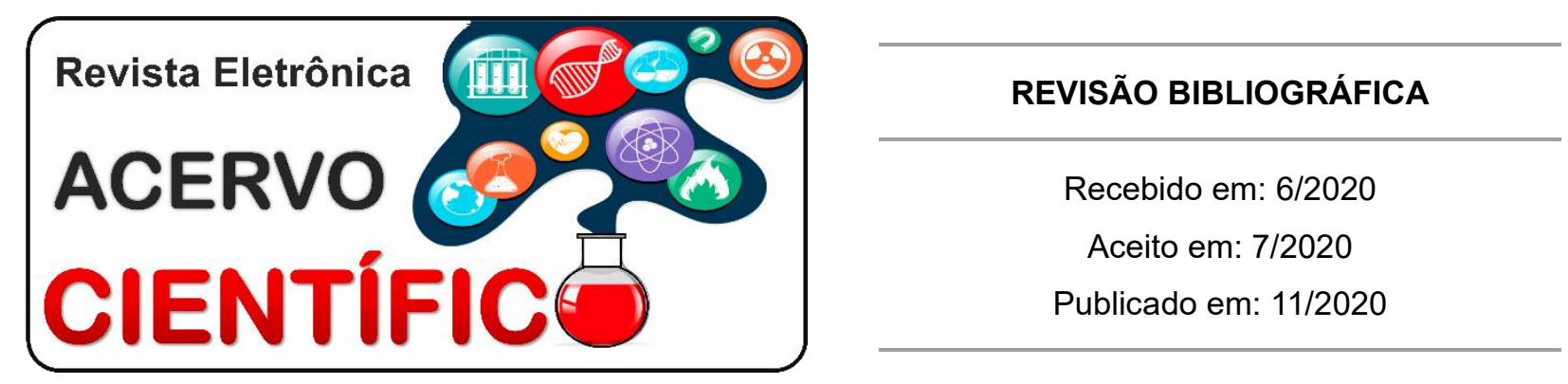

\title{
Fatores que interferem na qualidade da assistência ao parto humanizado
}

\author{
Factors that interfere with the quality of humanized childbirth care
}

\section{Factores que interfieren con la calidad de la atención humanizada del parto}

Rafael Radison Coimbra Pereira da Silva ${ }^{1 *}$, Juliana do Nascimento Sousa ${ }^{1}$, Paulo Roberto Pereira Borges $^{1}$, laggo Henrique de Sousa Figueiredo ${ }^{1}$, Thaysla de Oliveira Sousa ${ }^{2}$, Lorenna Maria Coêlho do Nascimento², Luana Rocha Cabral$^{2}$, Francisca Mikaelly Araújo do Nascimento², Paloma Esterfanny Cardoso Pereira ${ }^{3}$, Rauena Tágila Silva ${ }^{3}$.

Resumo: Esse artigo buscou analisar e descrever as principais dificuldades que os profissionais envolvidos na assistência ao parto se deparam na prestação da assistência humanizada. Este estudo trata-se de uma revisão de literatura realizada no período de Janeiro a fevereiro de 2020. Os dados coletados estavam presentes em meios eletrônicos de base de dados, especialmente, a Biblioteca virtual de Saúde (BVS), a Scientific Eletronic Library Online (Scielo) e a PUBMED. Foram utilizados os descritores: "parto humanizado", "assistência ao parto" e "obstetrícia" de forma única ou combinada. Evidenciou-se que apesar do parto humanizado ter ganhado maior destaque nos últimos anos, existem alguns fatores que dificultam que os profissionais utilizem dessas técnicas como a falta de estrutura e/ou materiais nas maternidades. Dessa forma, para que a assistência humanizada ao parto ocorra de forma eficaz e facilitada, se faz necessária uma reorganização nas maternidades a fim de proporcionar um ambiente acolhedor, espera-se por parte dos profissionais de saúde um atendimento mais solidário, além de medidas e procedimentos benéficos para 0 acompanhamento do parto e nascimento.

Palavras-chave: Parto humanizado, Assistência ao parto, Obstetrícia.

\begin{abstract}
This article sought to analyze and describe the main difficulties that professionals involved in childbirth care face in providing humanized care. This study is a literature review carried out from January to February 2020. The data collected were present in electronic means of the database, especially the Virtual Health Library (VHL), the Scientific Electronic Library Online (Scielo) and PUBMED. The descriptors were used: "humanized delivery", "delivery assistance" and "obstetrics" in a single or combined way. It became evident that although humanized childbirth has gained more prominence in recent years, there are some factors that make it difficult for professionals to use these techniques, such as the lack of structure and / or materials in maternity hospitals. Thus, for humanized childbirth assistance to occur effectively and facilitated, a reorganization of maternity hospitals is necessary in order to provide a welcoming environment, health professionals are expected to provide more supportive care, in addition to measures and beneficial procedures for monitoring delivery and birth.
\end{abstract}

Keywords: Humanized childbirth, Childbirth assistance, Obstetrics.

\footnotetext{
1 Universidade Estadual do Piauí (UESPI), Teresina - PI. *E-mail: rafaelcoimbra739@gmail.com

2 Faculdade Estácio (CEUT), Teresina - PI.

${ }^{3}$ Centro Universitário Santo Agostinho (UNIFSA), Teresina - PI.
} 
Resumen: Este artículo buscó analizar y describir las principales dificultades que enfrentan los profesionales involucrados en el cuidado del parto para brindar una atención humanizada. Este estudio es una revisión de la literatura realizada de enero a febrero de 2020. Los datos recolectados estuvieron presentes en los medios electrónicos de la base de datos, especialmente la Biblioteca Virtual en Salud (BVS), la Biblioteca Científica Electrónica en Línea (Scielo) y PUBMED. Se utilizaron los descriptores: "parto humanizado", "asistencia al parto" y "obstetricia" de forma única o combinada. Se evidenció que si bien el parto humanizado ha ganado más protagonismo en los últimos años, existen algunos factores que dificultan el uso de estas técnicas por parte de los profesionales, como la falta de estructura y / o materiales en las maternidades. Por lo tanto, para que la asistencia humanizada al parto ocurra de manera efectiva y facilitada, es necesaria una reorganización de las maternidades para brindar un ambiente acogedor, se espera que los profesionales de la salud brinden más atención de apoyo, además de medidas y procedimientos beneficiosos para controlar el parto y el nacimiento.

Palabras clave: Parto humanizado, Asistencia para el parto, Obstetricia.

\section{INTRODUÇÃO}

No Brasil, a assistência obstétrica consolidou-se no modelo tecnocrático e aos poucos ocorreu a institucionalização do parto, com excesso de medicalização, uso excessivo de tecnologia e despersonalização do cuidado colocando a mulher como paciente nesse processo (SILVA ALA, et al., 2017).

O parto passou a ser visto como processo patológico, de caráter mecanicista, em que necessita de intervenções e por sua vez e incorporando cada vez mais por procedimentos e técnicas invasivas e muitas vezes realizadas sem o consentimento da parturiente. O Brasil é atualmente um dos países com as maiores taxas de cesarianas. Uma média de $46,6 \%$ de cesarianas realizada, por ano. E na rede privada, essa taxa pode chegar a $85 \%$. Reflexo disso, o Brasil apresentou, em 2015, 62 casos de morte materna a cada 100 mil nascimentos (VARGENS OMC, et al., 2017).

Cada cesariana despropositada significa um risco maior de problemas, como infecções, hemorragia e complicações anestésicas, as quais auxiliam para o aumento das taxas de mortalidade materna. Para o bebê, o risco central relaciona-se aos problemas respiratórios causados pela a prematuridade, frequentemente correlacionadas com a remoção antecipada prévia do concepto antes da maturidade pulmonar plena. Além do mais, a quantidade de processos cirúrgicos, internações e tratamentos de complicações, significa a dilapidação de milhões de reais por ano aos cofres públicos no Sistema Único de Saúde (SUS). Estudos apontam que o parto cesariano tem despesas de duas a três vezes maiores que o parto normal (PRISZKULNIK G, CARRERO MA, 2009).

Dessa forma, a maioria das mortes maternas teriam como ser evitadas devido a diminuição das taxas de cesarianas, com o aperfeiçoamento na qualidade da atenção obstétrica nas atividades de saúde, no fomento ao parto vaginal e, em especial, com a introdução do parto humanizado nas atividades de saúde (PRISZKULNIK G, CARRERO MA, 2009).

O termo "parto humanizado" ganhou bastante destaque nos últimos anos e ainda é erroneamente interpretado por muitos como parto sem anestesia, ou apenas parto normal, no entanto o parto humanizado não deve ser entendido como um tipo de parto. A humanização do parto é um processo que visa desenvolver práticas e desincorporar condutas invasivas desnecessárias através de evidências científicas (MEDEIROS RMK, et al., 2016). Exclusivamente, durante o acolhimento ao parto, as ações propostas pelo Ministério da Saúde encontram-se baseadas nas instruções ao parto normal da Organização Mundial da Saúde (OMS). Desta forma, as condutas acrescentadas no parto normal são definidas em quatro categorias: procedimentos claramente úteis e que precisam ser estimuladas; procedimentos prejudiciais ou ineficientes e que devem ser abolidos; práticas com indícios falhos para sustentar uma orientação e que precisariam ser aplicadas com prudência; práticas regularmente empregadas de maneira inadequada, ocasionando mais desvantagens que melhorias (BUSANELLO J, et al., 2017).

Seguindo as recomendações da OMS para o implemento da humanização da assistência ao parto, foi lançado no Brasil, no ano 2000, o Programa de Humanização no Pré-natal e Nascimento (PHPN). Investindo 
na inclusão das enfermeiras Obstétricas no cuidado direto ao parto, visando combater os altos índices de mortalidade materna e violência obstétrica do país (BRASIL MS, 2000).

Com a implementação da Rede Cegonha a partir de 2011, instituída pelo Ministério da Saúde (MS) no âmbito do Sistema Único de Saúde (SUS), e a significativa participação dos enfermeiros obstétricos, em muitas maternidades, que até então não contavam com esses profissionais houve uma redução do número de cesarianas, mas ainda são observadas taxas muito acima dos $15 \%$ recomendados pela OMS (VARGENS OMC, et al., 2017). Esse acréscimo na quantidade de cesáreas em todo o mundo encontra-se associado à melhora no alcance das mulheres a esse processo em acontecimento de emergência, da mesma forma à prática indiscriminada sem recomendação médica. Cesáreas efetuadas de forma seguras e adequadas, seguindo um direcionamento médico organizado, são mecanismos fortemente salvadores de vidas. Porém, no entanto, a distribuição de cesarianas seguras e apropriadas ainda é uma grande dificuldade em países que tenham grande mortalidade materna, tornando-se escassos; de outra forma, sua quantidade em certas localidades faz com que a grande dificuldade ocasione em reduzir a produção de cesarianas sem recomendação clínica (MASCARELLO KC, et al., 2017).

A temática se faz interessante porque a filosofia do parto humanizado surgiu diante da violência obstétrica, e os profissionais empenhados em dar uma assistência de qualidade, buscam aperfeiçoar-se. Partindo dessa explanação, o estudo teve o objetivo de verificar na literatura quais as queixas identificadas pelos profissionais da área em relação a implementação da assistência humanizada ao parto.

\section{MÉTODOS}

Este estudo trata-se de uma revisão de literatura realizada no período de Dezembro de 2019 a Maio de $2020 \mathrm{com}$ base em artigos selecionados em que destacou-se a assistência ao parto humanizado e as dificuldades encontradas pelos profissionais de saúde inerentes a prestação deste cuidado.

Os dados coletados estavam presentes em meios eletrônicos encontrados por meio de pesquisa realizada nas seguintes bases de dados: Scientific Eletronic Library Online (Scielo), Biblioteca Virtual em Saúde (BVS) e Pubmed através dos descritores "parto humanizado", "assistência ao parto" e "obstetrícia" de forma única ou combinada.

Os critérios de inclusão adotados foram: artigos e teses em português, inglês e espanhol que disponibilizassem o texto completo em suporte eletrônico. Já como critérios de exclusão: livros, anais de congressos ou conferências e relatórios. Sendo incluídos artigos entre os anos de 2009 a 2017. Além disso, foram analisadas algumas portarias do Programa de humanização do pré-natal e nascimento (PHNP). (Figura 1). A amostra deu-se a partir da leitura do resumo dos artigos encontrados e seleção daqueles que responderam ao problema da pesquisa, totalizando 15 artigos, 3 portarias e 1 tese de mestrado. Para análise de conteúdo efetuou-se leitura integral e exaustiva dos artigos, para transcrição dos resultados e trechos significativos.

Figura 1 - Fluxograma metodologia.



Fonte: Silva RRCP, et al., 2020. 


\section{RESULTADOS E DISCUSSÃO}

Selecionaram-se 15 artigos, os quais serviram como base para o estudo literário. Desses, 8 estudos evidenciaram os principais fatores que dificultam a realização da assistência ao parto, segundo profissionais da saúde. São eles: Resistência dos profissionais de saúde, carência de leitos, tempo maior que exige a assistência humanizada, falta de materiais, falta de capacitação da equipe, poucos profissionais, falta de preparo das gestantes e rotina voltada aos profissionais da saúde (Quadro 1).

Quadro 1 - Síntese dos resultados dos estudos selecionados.

\begin{tabular}{|c|c|c|}
\hline Autor e ano & Objetivo & Principais resultados \\
\hline $\begin{array}{l}\text { ALMEIDA SMO, } \\
\text { SILVEIRA MFA, } 2009 .\end{array}$ & $\begin{array}{l}\text { Identificar os avanços e dificuldades } \\
\text { enfrentadas para implantação e } \\
\text { prática da humanização do parto. }\end{array}$ & $\begin{array}{c}\text { Resistência dos profissionais de } \\
\text { saúde, Alta demanda de pacientes } \\
\text { em relação a quantidade de leitos e } \\
\text { profissionais, maior tempo } \\
\text { necessário para o atendimento } \\
\text { humanizado, falta de materiais e } \\
\text { falta de capacitação da equipe. }\end{array}$ \\
\hline $\begin{array}{c}\text { ANDRADE LO, et al., } \\
2017 .\end{array}$ & $\begin{array}{c}\text { Conhecer como são desenvolvidas } \\
\text { as práticas de humanização durante } \\
\text { o trabalho de parto. }\end{array}$ & $\begin{array}{l}\text { Falta de conhecimento das } \\
\text { gestantes, equipe reduzida e } \\
\text { resistência dos profissionais. }\end{array}$ \\
\hline $\begin{array}{l}\text { BUSANELLO J, et al., } \\
2011 .\end{array}$ & $\begin{array}{l}\text { Analisar as práticas desenvolvidas } \\
\text { na assistência à adolescente, a partir } \\
\text { do relato dos trabalhadores de um } \\
\text { Centro Obstétrico de um Hospital } \\
\text { Universitário, com base na proposta } \\
\text { de humanização do parto do } \\
\text { Ministério da Saúde. }\end{array}$ & $\begin{array}{l}\text { Estrutura física inadequada do } \\
\text { Centro Obstétrico; a falta de postura } \\
\text { dos trabalhadores da saúde acerca } \\
\text { das particularidades de cada } \\
\text { parturiente; a falta de preparo e } \\
\text { conhecimento das parturientes e dos } \\
\text { familiares para o enfrentamento do } \\
\text { processo de parturição. }\end{array}$ \\
\hline CAMILO AR, et al., 2013. & $\begin{array}{l}\text { Problematizar as causas que } \\
\text { interferem no processo de } \\
\text { assistência de enfermagem } \\
\text { humanizada no parto normal. }\end{array}$ & $\begin{array}{l}\text { Carência de leitos e de unidades } \\
\text { assistenciais; estrutura física } \\
\text { inadequada; falta de capacitação dos } \\
\text { profissionais e o modelo institucional. }\end{array}$ \\
\hline DODOU HD, et al., 2017. & $\begin{array}{c}\text { Conhecer a percepção de } \\
\text { profissionais da saúde sobre o } \\
\text { ambiente de trabalho da sala de } \\
\text { parto e sua interface com a } \\
\text { humanização da assistência. }\end{array}$ & $\begin{array}{l}\text { estrutura física deficiente, falta de } \\
\text { materiais e manutenção de } \\
\text { equipamentos, competência dos } \\
\text { profissionais e falta de integração da } \\
\text { equipe. }\end{array}$ \\
\hline SILVA ALA, et al., 2017. & $\begin{array}{l}\text { Avaliou a qualidade da atenção ao } \\
\text { parto na rede pública hospitalar, na } \\
\text { cidade do Recife, Pernambuco, } \\
\text { Brasil, segundo satisfação das } \\
\text { gestantes. }\end{array}$ & $\begin{array}{c}\text { Falta de conexão entre os serviços } \\
\text { que prestam assistência pré-natal e } \\
\text { aqueles que disponibilizam a } \\
\text { assistência ao parto, carência e má } \\
\text { distribuição de vagas e leitos } \\
\text { obstétricos. }\end{array}$ \\
\hline SILVA LNM, et al., 2017. & $\begin{array}{l}\text { Discutir os aspectos institucionais, a } \\
\text { partir de uma perspectiva dinâmica e } \\
\text { interrelacional, nos limites e } \\
\text { possibilidades para a } \\
\text { operacionalização da Proposta de } \\
\text { Humanização da Atenção ao Parto e } \\
\text { Nascimento. }\end{array}$ & $\begin{array}{l}\text { Problemas estruturais; alta demanda } \\
\text { de pacientes; poucos materias e } \\
\text { equipamentos necessários; }\end{array}$ \\
\hline SOUZA TG, et al., 2011. & $\begin{array}{l}\text { Conhecer a percepção dos } \\
\text { profissionais de saúde que atuam na } \\
\text { assistência ao parto sobre a } \\
\text { humanização do processo de } \\
\text { nascimento. }\end{array}$ & $\begin{array}{c}\text { Normas e rotinas hospitalares e } \\
\text { inadequado espaço físico das salas } \\
\text { de parto e pré-parto. }\end{array}$ \\
\hline
\end{tabular}

Fonte: Silva RRCP, et al., 2020. 
Constatou-se que a assistência humanizada ao parto começa bem antes do parto propriamente dito, no pré-natal, onde deve ser feito o acompanhamento da gestante e se for o caso também com o companheiro dela no pré-natal masculino. Portanto, para a efetivação da Humanização no atendimento o profissional deve oferecer assistência a gestante e ao parceiro realizando um conjunto de condutas, atitudes e posturas, ausentes de julgamentos e baseadas no diálogo, empatia e acolhimento, promovendo a autonomia e o protagonismo de mulheres durante a gravidez e parturição, fazendo consultas de pré-natal e exames, além de fornecer informações e esclarecimento de dúvidas, preparando-os assim para o momento do parto e puerpério (ANDRADE LO, et al., 2017).

Em 2003, foi criada a Política Nacional de Humanização (PNH), popularmente conhecida como Humaniza SUS, o objetivo de sua criação e implantação nos serviços de saúde em linhas gerais pauta-se na diminuição das filas e tempo de espera, atendimento acolhedor e resolutivo e garantia de direitos dos usuários. Além disso, ela tem diretrizes (Acolhimento, ambiência, clínica ampliada e compartilhada, gestão participativa e cogestão, valorização do trabalhador e defesa dos direitos dos usuários) e princípios (Transversalidade, indissociabilidade entre atenção e gestão, Protagonismo, corresponsabilidade e autonomia dos sujeitos e coletivos). Diante do exposto, observa-se que a criação da PNH foi embasada na tentativa de minimizar os impactos causados pelo modelo tecnocrático dando espaço para uma assistência mais humana e que coloca a mulher como protagonista do próprio parto (BRASIL MS, 2006).

Todavia, o termo humanização para o grupo de gestantes, parturientes e puérperas foi usando bem antes de 2003, em 2000, no Programa Humanização do Pré-Natal e Nascimento, através da Portaria GM/MS nำ569, de $1^{\circ}$ de junho de 2000, que também afirma que o atendimento digno e de qualidade a gestante e aos recém-nascidos são direitos inalienáveis a cidadania, a portaria foi lançada mediante a necessidade de medidas complementares que visassem a diminuição da morbimortalidade materno, perinatal e neonatal no Brasil (BRASIL MS, 2000).

Evidenciou-se que os problemas encontrados para realizar o atendimento humanizado são principalmente relacionados aos limites impostos pelas estruturas físicas encontradas nas maternidades e/ou rotinas hospitalares. A grande demanda de pacientes torna-se um empecilho devido os poucos profissionais e leitos disponíveis nas maternidades somado ainda com falta de materiais e maior tempo que leva o atendimento humanizado faz com que os profissionais sejam poucos adeptos da prática (ALMEIDA SMO, SILVEIRA MFO, 2009).

Souza TG, et al. (2011) trazem em seus resultados e discussões diversas falas de profissionais que trabalham em maternidades onde é possível constatar que uma parte dos profissionais tentam ou tentaram fazer com que $o$ atendimento fosse mais humanizado, entretanto acabaram se frustrando ao encontrarem barreiras como: desinteresse da equipe, carência de leitos, poucos profissionais e até despreparo da família e/ou da gestante. Em contrapartida, existem relatos positivos, onde os profissionais e as instituições conseguem realizar um excelente atendimento mesmo com limitações institucionais.

Ademais, pode-se notar que o modelo tecnocrático se mostrou um dos grandes limitadores da humanização do parto. A rotina hospitalar voltada aos profissionais acaba diminuindo o protagonismo e a autonomia da gestante, isto, somado a falta de capacitação e consequente desinteresse da equipe exercem influência direta na efetivação ou não da assistência humanizada ao parto. Logo, algumas estratégias podem ser lançadas na tentativa de reduzir o impacto do modelo tecnocrático, sendo elas: contratação de mais profissionais, melhor dimensionamento da equipe, implantação de educação em saúde continuada e oferta de cursos de capacitação (CAMILO AR, et al., 2012).

Diante do exposto, torna-se válido ressaltar que existem diversas formas de humanizar o parto e mesmo com estrutura física inadequada, com interesse e um bom dimensionamento da equipe é capaz de humanizarse toda a assistência. Levando em consideração o conceito de humanizar, já explanado aqui, medidas como: projetos de ensino voltados as gestantes, acolhimento embasado no respeito, garantia dos direitos da gestante e posicionamento de parir mais confortável para a mulher são algumas das formas de tornar o "parir" menos tecnicista e garantir a autonomia da mulher tornando desde a gestação até o parto mais humano. Entre as várias técnicas humanística existentes atualmente para o momento do parto propriamente dito, 
ressaltamos algumas formas não farmacológicas de alívio da dor encontradas durante a pesquisa e que têm eficácia comprovadas cientificamente. Sendo elas: musicoterapia, hidroterapia, rebozo, massoterapia, hipnose, aromaterapia e até o próprio posicionamento na hora de parir, já mencionado aqui. Visto o que humanizar é, o profissional deve deixar a parturiente confortável, respeitando suas escolhas, a dor, sentimentos, e acima de tudo acolher, cuidar e, sobretudo, saber ofertar atenção (MAFETONI RR E SHIMO AKK, 2014). Por conseguinte, se observa que para realização de um atendimento humano e acolhedor é necessário ter uma equipe multiprofissional que os mesmo possuam tempo disponível para exercer a pratica humanizada, protagonizando a parturiente e enfatizando o tempo correto para o nascimento do bebê, utilizando técnicas para o alívio de dores, e assim e consiga dar assistência qualificada e completa. Porém isso não é uma realidade nas maternidades aonde o número de parturientes e superior ao número de leitos e profissionais, contribuindo para uma assistência não qualificada, mas que pode ser minimizado com um bom dimensionamento de equipe.

O modelo tecnocrático afasta a mulher do seu próprio parto, ou seja, retira-a como a protagonista desse acontecimento, considera a gestação como algo inseguro, requisitado de múltiplas intervenções, muitas vezes desnecessárias e prejudiciais para mãe e bebê. Além disso, esse modelo de atenção relaciona o aumento da tecnologia com qualidade para o processo parturitivo, sendo que as cirurgias de cesariana acontecem em mais de $82 \%$ dos partos na rede privada. A cesariana quando é necessária, salva vidas. Todavia em gestações de baixo risco e sem complicações, a indicação da Organização Mundial da Saúde (OMS) é o parto vaginal, pois ainda que realizada com frequência, a cirurgia de cesariana apresenta inúmeros riscos de mortalidade e morbidade para mãe e bebê. Coloca-se o profissional como centro, ao invés da parturiente e sua família. Uma pesquisa realizada em Recife, em 2014 com 603 puérperas revelou índices bastante altos com relação as intervenções (86,5\%) sofreu algum tipo de intervenção durante o parto. Algumas intervenções comuns são a manobra de kristeller (37\%), a episiotomia (56\%) e uso de ocitocina de rotina e amniotomia (40\%) (SOUZA KJ, 2014).

Dados estatísticos representam que as mulheres brasileiras em torno de um quarto das que tiveram parto normal relataram sofrer algum tipo de violência ou maus tratos durante o tempo que estiveram na maternidade. Diante disso, analisada o conteúdo da violência obstétrica, existe a carência de mudanças dessa prática, com orientações aos assistentes incluindo alterações no ambiente e no modo de trabalho daqueles profissionais de saúde como o próprio enfermeiro. Por esse motivo foram criadas como estratégias a aplicação da rede cegonha pelo Ministério da Saúde com o objetivo de fornecer um atendimento totalmente humanizado para as gestantes diminuindo assim, a mortalidade materna e neonatal, respeitando a escolha do planejamento reprodutivo, com humanização do parto atenciosa e pontos associados ao aborto e puerpério (MEDEIROS RMK, et al., 2016).

Dessa forma, se observa que para humanizar o trabalho de parto, existe uma necessidade tanto de conhecimento e comprometimento da equipe para realizar os procedimentos, mas, principalmente, da gestão hospitalar para fazer um dimensionamento justo para minimizar sobrecargas e buscar aumentar os insumos para fornecimento de subsidio para o trabalho da equipe de saúde em atendimento, e assim obter resultados positivos no atendimento (DODOU HD, et al., 2017).

A carga simbólica que o ambiente da maternidade ocasiona na vida das mulheres são construtos de um misto de realização e insegurança, decorrente de uma série de violações de direitos. Toda mulher brasileira tem garantido pela Lei de Humanização do Parto direito a escolher os procedimentos que serão realizados em seu corpo; a ter acesso a informações verdadeiras e confiáveis; a ter respeito ao seu momento vivido, de ter contato com o seu filho após o parto; e de ter um acompanhante em todo o processo (SOUZA TG, et al., 2011). No âmbito dos serviços de saúde, a violência institucional é aquela em que há a reprodução das desigualdades nas relações de poder presentes no espaço social para o âmbito hospitalar. As regras institucionais são importantes para a organização dos serviços de saúde, no entanto, o privilégio exclusivo das normas e rotinas em detrimento das necessidades das parturientes configura uma assistência obstétrica que desfavorece a autonomia, o respeito e a dignidade das mulheres e constitui uma violência institucional perpetrada pelos serviços de saúde (SOUZA JK, et al., 2014). 


\section{CONSIDERAÇÕES FINAIS}

O conceito de humanização do parto estar relacionado a garantia dos direitos, autonomia e protagonismo da gestante e deve começar desde o pré-natal, onde deve-se oferecer um acolhimento holístico e integral, baseado no respeito e empatia. Possibilitando assim uma melhor compreensão das necessidades da parturiente. O pré-natal configura-se assim uma importante ferramenta no âmbito da humanização, pois, é nesse momento onde será esclarecido e sanado muitas dúvidas além de todo o acompanhamento profissional qualificado necessário para que uma gestação corra da melhor forma possível. Deve-se informar a paciente todos os seus direitos, para garantir a humanização. Infelizmente, fatores como estrutura física inadequada, leitos insuficientes, e uma demanda de pacientes superior as de profissionais funcionam como limitadores da humanização, dessa forma, para a efetiva implantação da assistência humanizada do parto é preciso resolver algumas problemáticas, em sua maioria estruturais e gerenciais. Dessa forma, faz-se necessário uma reorganização nas maternidades a fim de proporcionar as gestantes um ambiente acolhedor, empático e sobretudo respeitoso. Espera-se que este trabalho sirva como fomento para novas pesquisas acerca do assunto e que possa contribuir na busca de possíveis soluções para uma maior qualidade na assistência ao parto humanizado.

\section{REFERÊNCIAS}

1. ALMEIDA SMO, SILVEIRA MFA. Humanizing delivery: progress and difficulties for its implementation. Revista de enfermagem da UFPE online, 2009; 3(4): 945-952.

2. ANDRADE LO, et al. Práticas dos profissionais de enfermagem diante do parto humanizado. Revista de enfermagem da UFPE online, 2017; 11(6): 2576-2585.

3. BRASIL MS. Portaria n.ำ 1,459 de 24 de junho de 2011. Institui no âmbito do Sistema Único de Saúde (SUS): a Rede Cegonha. Diário Oficial da União, jun. 2011.

4. BRASIL MS. Secretaria de Atenção à Saúde. Núcleo Técnico da Política Nacional de Humanização. Humaniza SUS: documento base para gestores e trabalhadores do SUS. 3.ed. Brasília: Ministério da Saúde, 2006.

5. BRASIL MS. Secretaria de Políticas de Saúde. Programa de humanização no pré-natal e nascimento. Brasília, 2000.

6. BUSANELLO J, et al. Atenção humanizada ao parto de adolescentes: análise das práticas desenvolvidas em um centro obstétrico. Revista Brasileira de enfermagem, Brasília. 2011; 64 (5): 824-832.

7. CAMILO AR, et al. Aspectos que dificultam assistência humanizada ao parto normal. Revista de trabalhos acadêmicos, Campus Niterói, 2013; 4(6): 1-8.

8. CARNEIRO LMA, et al. Parto natural X parto cirúrgico: percepções de mulheres que vivenciaram os dois momentos. Revista de Enfermagem do Centro-Oeste Mineiro, 2015; 5(2): 1574-1575.

9. DODOU HD, et al. Sala de parto: condições de trabalho e humanização da assistência. Caderno de Saúde Coletiva, 2017; 3(25): 332-338.

10. MAFETONI RR, SHIMO AKK. Métodos não farmacológicos para alívio da dor no trabalho de parto: revisão integrativa. Revista Mineira de Enfermagem, 2014; 18(2): 505-520.

11. MASCARELLO KC, et al. Complicações maternas e cesárea sem indicação: revisão sistemática e meta-análise. Revista de Saúde Publica, 2017; 51-105.

12. MEDEIROS RMK, et al. Humanized Care: insertion of obstetric nurses in a teaching hospital. Revista Brasileira de Enfermagem, 2016; 69(6): 1091-1098.

13. PRISZKULNIK G, CARRERO MA. Parto humanizado: influências no segmento saúde. Mundo Saúde. 2009;33: 8088.

14. SILVA ALA, et al. A qualidade do atendimento ao parto na rede pública hospitalar em uma capital brasileira: a satisfação das gestantes. Cadernos de Saúde Pública, 2017; 33(12): 1678-4464.

15. SILVA ALS, et al. Práticas de enfermeiras para promoção da dignificação, participação e autonomia de mulheres no parto normal. Escola Anna Nery, 2015; 19(3): 424-431.

16. SILVA LNM, et al. Programa de humanização do parto e nascimento: aspectos institucionais na qualidade da assistência. Revista de Enfermagem da UFPE online, 2017; 11(8): 3290-3294.

17. SOUZA KJ. Violência institucional na atenção Obstétrica: proposta de modelo preditivo para depressão pós-parto. Dissertação (Mestrado em Saúde Coletiva) - Programa de pós-graduação em saúde coletiva. Universidade de Brasília, Brasília, 2014; 106 p.

18. SOUZA TG, et al. A humanização do nascimento: percepção dos profissionais de saúde que atuam na atenção ao parto. Revista Gaúcha de Enfermagem, 2011; 32(3): 479-486.

19. VARGENS OMC, et al. Contribuição de enfermeiras obstétricas para consolidação do parto humanizado em maternidades no Rio de Janeiro-Brasil. Escola Anna Nery, 2017; 21(1): 2177-9465. 